\title{
Applications of Radiodermatography to Human and Primate Studies
}

\author{
STANLEY M. GARN, ANDREW K. POZNANSKI AND JOHN C. GALL \\ Center for Human Growth and Development, the Departments of \\ Radiology and Pediatrics, University of Michigan, Ann Arbor and the \\ Plymouth State Home and Training School, Northville, Michigan
}

\begin{abstract}
Using 325-mesh tantalum powder as a contrast medium, it is possible to take high-resolution "radiodermatographs" of man and infra-human primates that combine the advantages of postero-anterior hand radiographs and conventional paper palm-and-finger prints. The radjodermatographs are particularly useful in relating creases, triradii and fingertip ridge patterns to differences in the proportions of the carpals, metacarpals and phalanges.
\end{abstract}

With the increasing use of dermatoglyphics in population comparisons and in the diagnosis of congenital disorders, there is need to relate palmar and digital creases and ridge patterns to differences in the underlying skeleton. Such a need also exists in primate studies, where the size and arrangement of the volar pads bear a relationship to the proportions of the round and tubular bones.

One approach to the problem has been to make separate radiographs and paper prints, using radiopaque markers for partial registry (Valsik, '28, cited in Cummins and Midlo, '61). Another approach is to employ radiopaque contrast material to fill the areas between the ridges (Béclère, '18; Castellanos, '39). However, fine-mesh tantalum powder, now commercially available, offers many advantages over previously used contrast materials. The sinteringgrade tantalum powder (Ciba Rare Chemicals Division, Summit, New Jersey) is highly radiopaque, yet non-toxic, a particular advantage when working with children and the mentally retarded. Tantalum-powder is the basis of the technique of "radiodermatography" which we have recently described (Poznanski, Gall and Garn, '69).

As shown in figure 1 , the tantalum powder defines both creases and ridge patterns and holds fine detail without obscuring the bone-shadows. Ridges appear as dark lines as in conventional prints. The transverse palm creases are visible over their entire length, digital and axial triradii can be related to the metacarpals, and the finger- prints are especially well defined. The second illustration (fig. 2) shows the practicality of subsequent photographic enlargement, and the fact that a larger surface is recorded via radiography than with inktype or inkless impressions.

Compared to figure 1 , the dermatoglyphic alterations in the Holt-Oram syndrome (fig. 3) demonstrate the clinical value of radiodermatography. In the example pictured, the individual has a non-opposable "thumb" with an extra segment, metacarpal elongation and numerous carpal, metacarpal and digital abnormalities. As shown in figure 3 , there is but a single major palm crease (the distal transverse crease). Further, all four digital triradii are distally displaced compared to their more usual locations. All palm ridge-patterns are atypical. In trisomy $\mathbf{G}$, by way of comparison, the digital triradii and the transverse creases tend to be proximally displaced and, with reduction of metacarpals $3-5$, may form a single transverse crease or fold midway between the metacarpal-phalangeal-creases and the bracelet creases.

Radiodermatography is also useful in primate research, especially since the radiographic images can be enlarged. As shown in figure 4 , the tantalum-powder contrast technique reveals the complex volar padmarkings in the rhesus monkey, as well as the location of pad patterns and creases relative to the ends of the tubular bones. Six palm-pads are identified and the details of the enlarged digital-tip pads are re- 


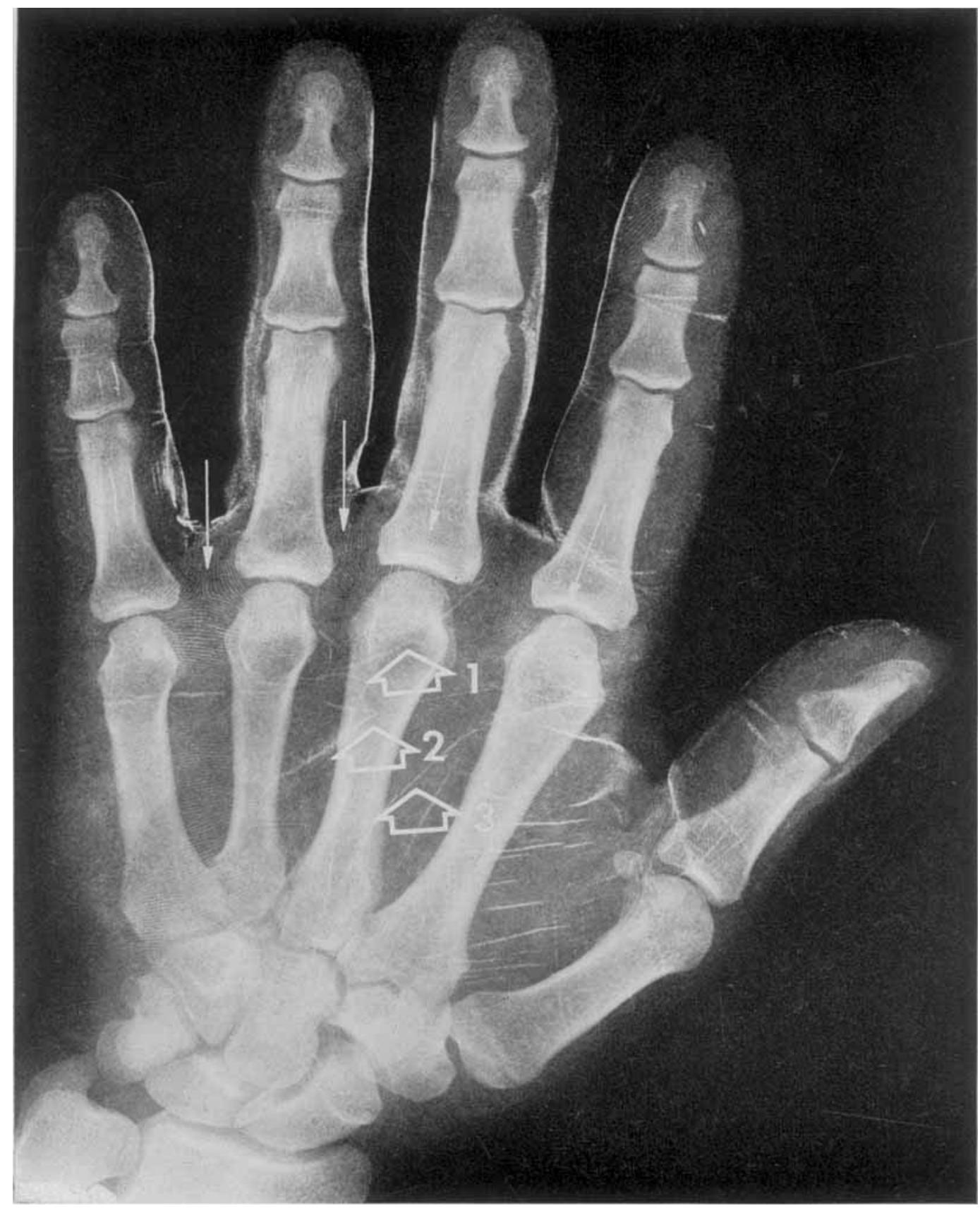

Fig. 1 Postero-anterior left hand medium-contrast "radiodermatograph" showing the digital triradii (tips of long arrows), the distal and proximal transverse creases (1 and 2) and the radial longitudinal crease ( 3 ) relative to the underlying bones. 


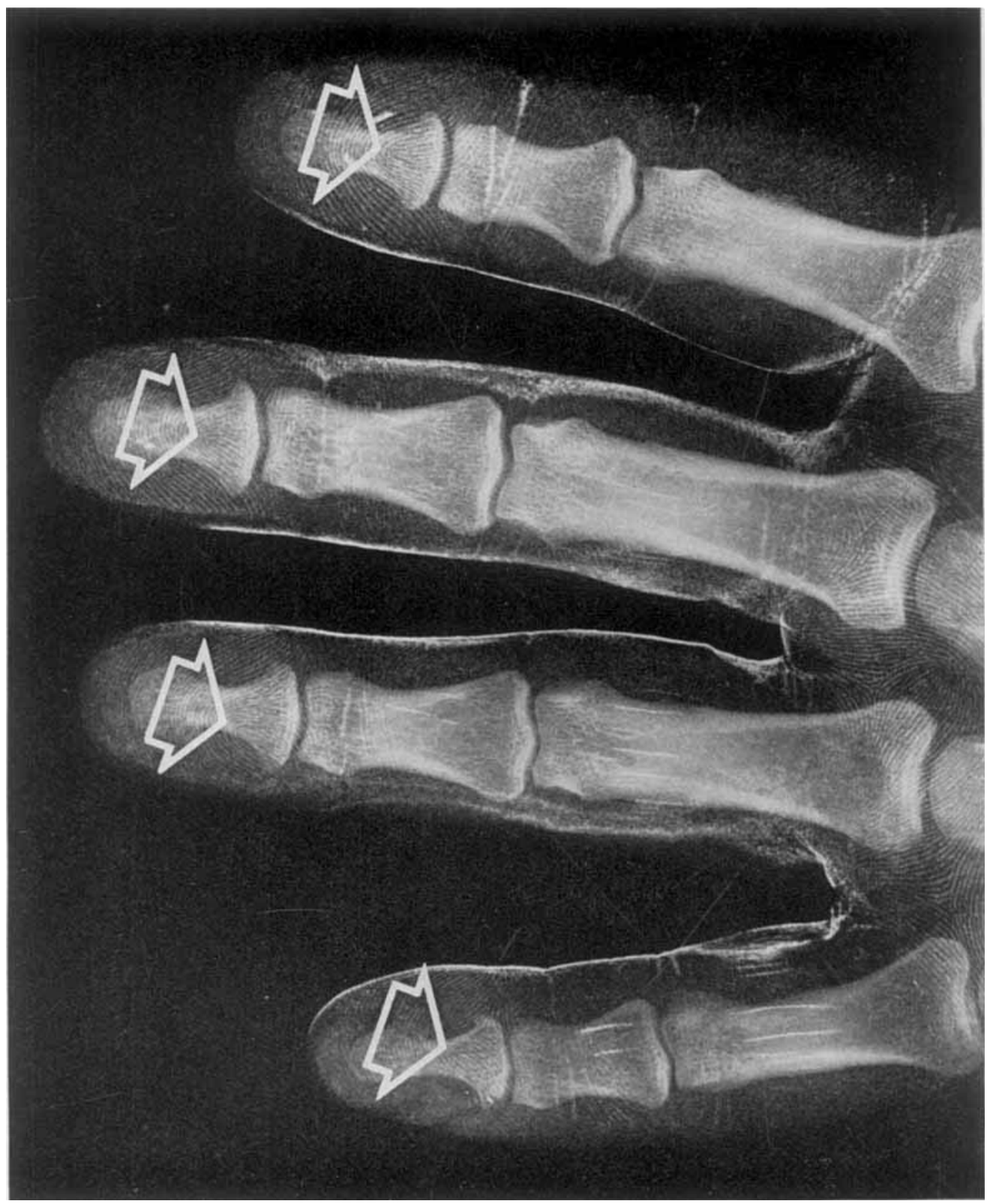

Fig. 2 Enlarged section of figure 1, showing loops on fingers 2-5. The tantalum-powder radiodermatograph reveals fingertip detail without rolling, the crease patterns and the triradii all in a single operation.

vealed without the need for "rolling." Furthermore, the films show excellent ridge detail, including incipient ridges and sweatgland openings, as in the enlarged section depicted in figure 5.
The technique of radiodermatography is otherwise conventional, with no increase in Peak Kilovoltage or Milliampere-seconds. Best results are obtained with non-screen films, either those intended for manual 


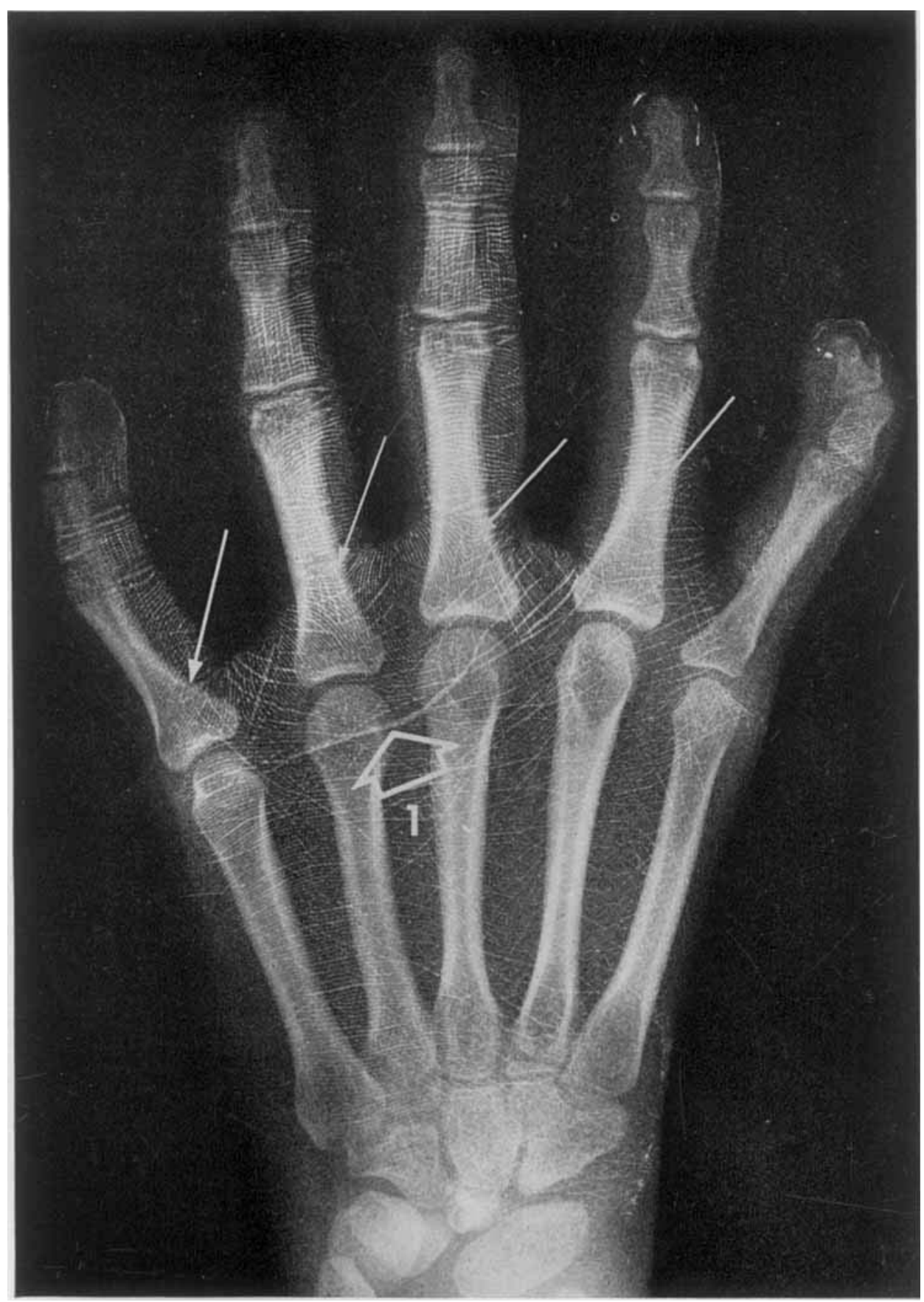

Fig. 3 Low-contrast radiodermatograph of a girl with the Holt-Oram syndrome. In this extreme case, only the distal palm crease (1) exists, and the digital triradii (indicated by arrows) are more distal in position than usual and the palm pattern is greatly altered from the normal. 


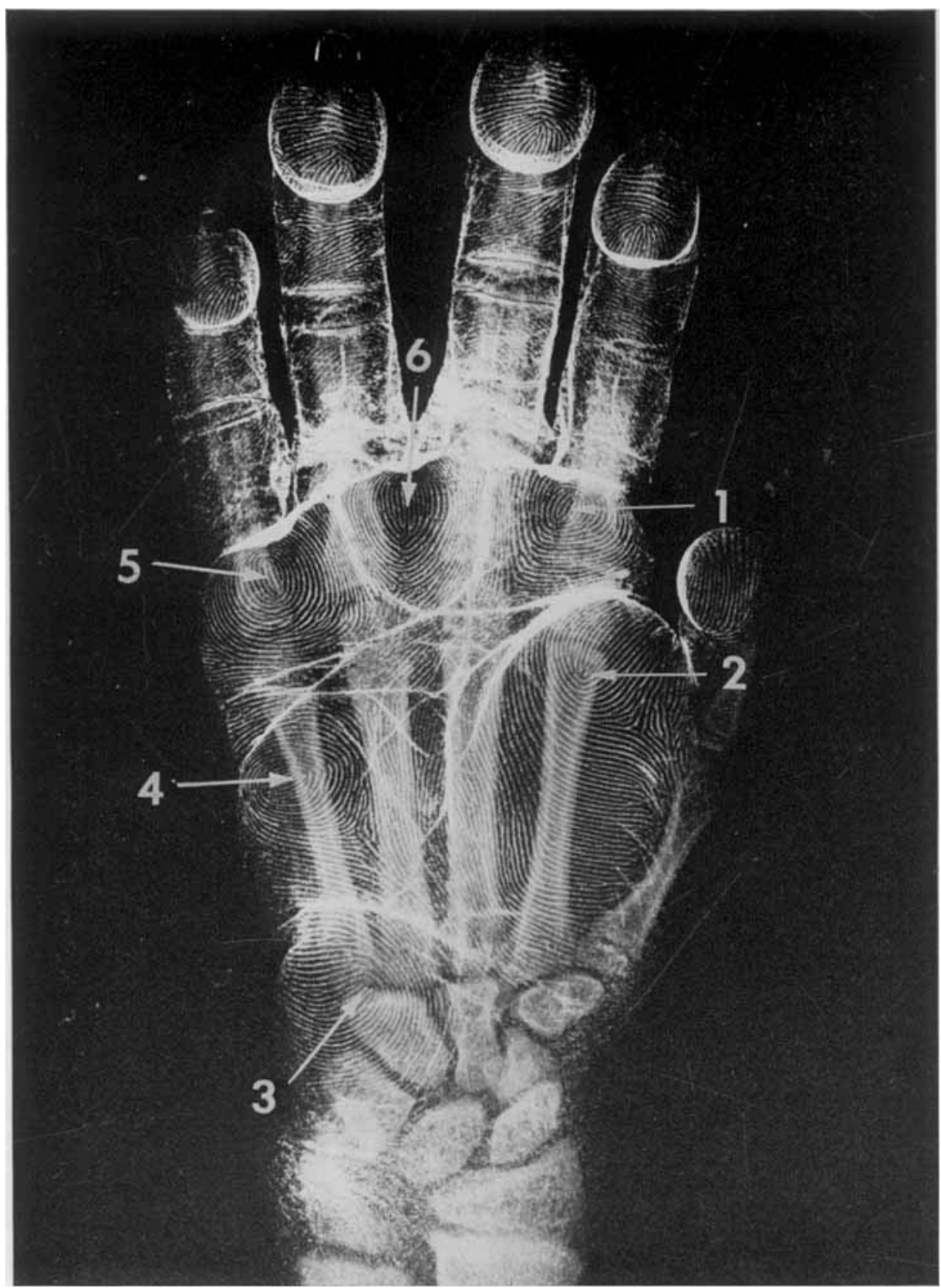

Fig. 4 High-contrast radiodermatograph of a young rhesus monkey showing the enlarged fingertip patterns, six volar-pad patterns (1-6) and well-defined palmar creases. Compare with the hand in figure 1.

processing or those developed for rapid automatic processing (R.P.) machines. For primates and for very small human hands, Kodak Mammography Film or industrial film may be used for even greater contrast. By altering exposure, film-type and processing time, contrast and density can be adjusted to favor crease-patterns, ridge-pat- 


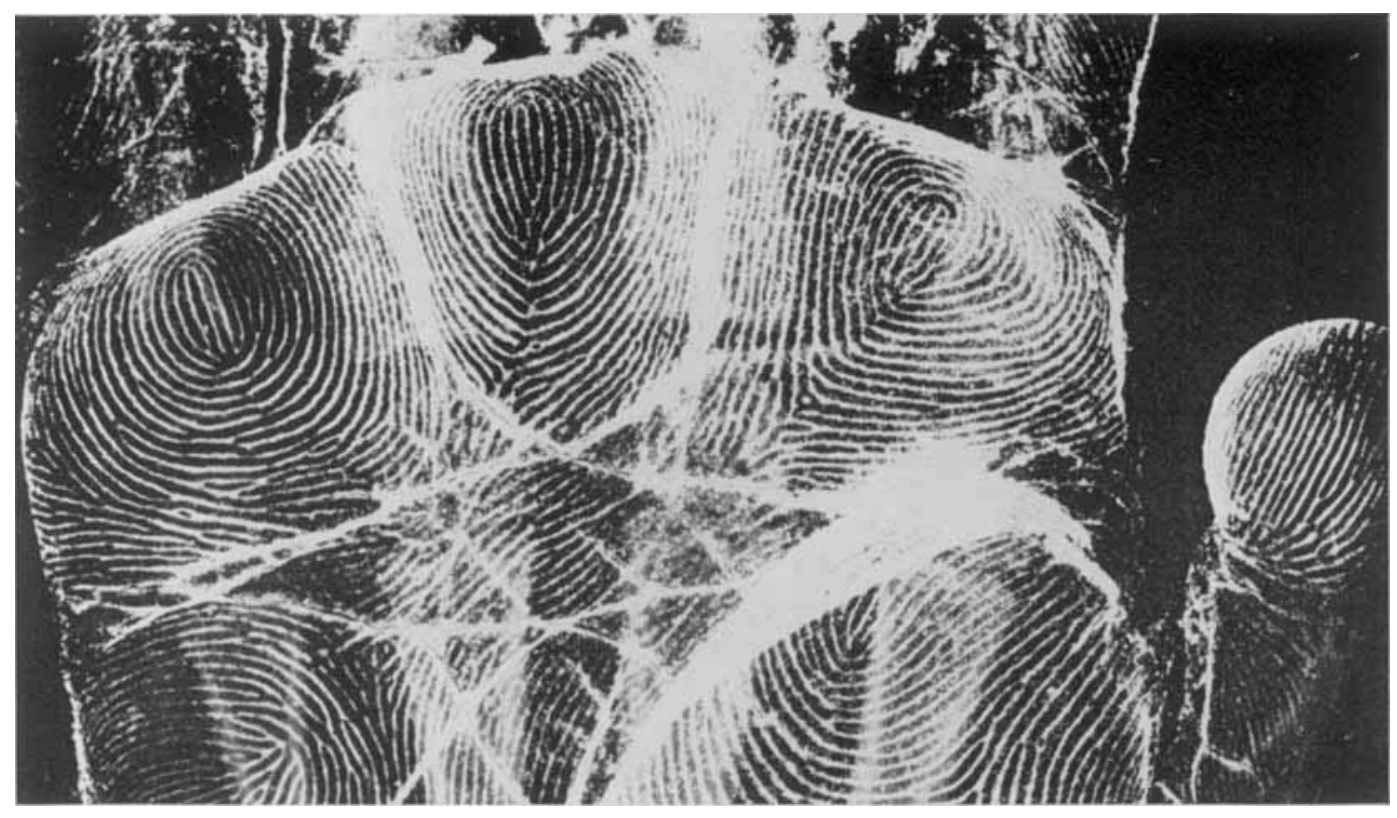

Fig. 5 Enlarged section of figure 4 showing pad-pattern detail, sweat-gland openings and incipient ridges. Since contact is not needed, the radiogr aphic projection can show morc detail than ink-type or inkless prints. Note that ridges appear dark, as in conventional "fingerprints."

terns or bone details. Radiodermatographs can also be employed for subperiosteal, medullary and cortical width measurements.

Besides the obvious advantage of obtaining finger-and-palm prints in exact registry with underlying structures, radiodermatographs have at least seven other investigative benefits. The need for "rolling" is eliminated, and the radiographic projections yield ridge detail over a larger area of the fingers. Pressure is not critical, and far more palm detail can be obtained. Resolution is high, and incipient ridges and sweat-gland openings can be identified and counted. Enlargements can be made without loss of detail due to paper texture. Multiple films can be made with a single exposure, or two different films can be combined to yield both high-contrast and lowcontrast radiodermatographs. Ridges remain dark and valleys light, so that previous experience with ink-prints does not re- quire re-training. Finally, card-punching, digital-readout measuring devices can be employed to simplify mensuration and maximize data-gathering in the combined print and bone-shadow radiodermatographs.

\section{ACKNOWLEDGMENT}

We appreciate the assistance of Dr. Jose C. Elgoyhen in making the rhesus radiodermatographs.

\section{LITERATURE CITED}

Béclère, M. H. 1918 La Radiographie Anthropométrique du Pouce. (Superposition des empreintes digitales, du squelette et de l'ongle). Comptes Rendus Acad. Sci., 167: 499-500.

Cummins, H., and C. Midlo 1961 Fingerprints, Palms and Soles. Dover Publications, New York.

Castellanos, I. 1939 Identification Problems, Criminal and Civil. R. V. Basuino, Brooklyn.

Poznanski, A. K., J. C. Gall and S. M. Garn 1969 Radiodermatography: Simultaneous demonstration of dermatoglyphics and osseous structures in the hand. Invest. Radiol., 4: 340-342. 